\title{
Digital Pattern Product Development: a pattern-driven approach for improving the bidding process in rail transport systems
}

\author{
D. Sanzari ${ }^{1}$, B. Brunetti ${ }^{2} \&$ V. Torrieri ${ }^{1}$ \\ ${ }^{I}$ Department of Civil, Architectural and Environmental Engineering, \\ Federico II University of Naples, Italy \\ ${ }^{2}$ Ansaldo STS S.p.A., Italy
}

\begin{abstract}
The planning and construction of a rail transportation system requires the implementation of several tasks where a major role is assumed by enterprises which have to pre-design, design and produce/build in detail any element of a rail system (i.e. infrastructure, signalling system and rolling stock). Currently, different divisions of a company perform different tasks or sub-tasks without adopting any (or sometimes only a partial) integrated approach in the various phases of product development. Our proposal, based on the case study of the multinational enterprise Ansaldo STS, is to develop a digital pattern approach for product development which consists of adopting an integrated approach in all phases of the industrial process in order to avoid redundancy and duplication in working phases, and lost times in redesign and in information retrieval. The proposed approach is based on a web platform which allows information sharing among the different phases of project implementation and data (know-how) concerning past projects. Finally, the approach is applied to design the signalling system for a conventional metro system.

Keywords: signalling system design, knowledge management, industrial process development, industrial product development.
\end{abstract}

\section{Introduction}

In recent decades, the planning of public transport systems has been considered a useful tool for re-balancing the modal split and reducing the use of private cars (as shown, for instance, by [1] and [2]). Indeed, especially in urban contexts, the 
extensive use of the road system generates negative externalities such as congestion, accidents, noise and air pollution [3]. Hence, local administrations generally adopt regulatory instruments such as pricing policies $[4,5]$ or restricted traffic zones [6] to limit the use of private cars.

With these assumptions, the development of a rail and/or metro system can be considered a useful backbone of a rational public transportation system. In this respect the process for planning and managing a rail system can be broken down into four phases:

- planning and defining project requirements;

- tender and bidding;

- detailed design and construction of infrastructure and vehicles;

- operational management of the transportation system.

The first phase, generally performed by local administrations, is based on implementing traditional proven techniques whose aims are to determine macrorequirements (such as routes, maximum frequencies, maximum number of passengers per convoy, etc.) to be put out to tender.

The second phase has two different kinds of actors: the local administration which puts out a tender for the construction of the rail system and one or more enterprises which have to pre-design the system in order to determine their manufacturing costs and hence respond positively with a bid.

The third phase, provided by the enterprise which wins the competition, consists of designing and producing/building in detail any element of the rail system (i.e. infrastructure, signalling system and rolling stock).

Finally, the last phase is implemented by the operator of the rail/metro system whose aim is to provide efficient and effective services of high quality.

The first phase has been analysed extensively in the literature (see, for instance, $[1,2,7]$ and [8]). Likewise, for the fourth phase, numerous studies have investigated the interaction between rail system performance and travel demand [9-11], the management and rescheduling of rail services in the event of perturbation [12-16] or the identification of optimal driving profiles which minimise energy consumption [17-20].

The focus of this paper is to analyse the improvement of the Digital Pattern Product Development approach in the case of the Italian multinational company Ansaldo STS (ASTS). The company is engaged in designing and building all technological elements by means of which all rail transport components (such as switches, signals, power stations, telecommunication systems and on-board computers) can be controlled. The management structure of the company is completely integrated and divided into three units: the Signalling Business Unit (SBU) which develops signalling system solutions, the Transportation Business Unit (TBU) which develops rail transport solutions, the Standard Platform and Products Unit (SPPU) which develops, according to regulations and ASTS standards, the tools that are used by the other operating units to design and check signalling and transportation solutions.

As described above, the production of a new signalling system or transport solution involves the task of preparing the bid (Bidding Process, BP) and 
implementation of the bid technical solution (Project Engineering Process, PEP). In particular, BP activities consist in a preliminary design of the integrated transport system, which involves: $(a)$ defining the terms of the bid solution within the constraints of specification, technical regulations and corporate standards; (b) identifying the technological gap that needs to be filled (if necessary); (c) maximising project profitability (i.e. the cost/benefit ratio). Likewise, the phases of the PEP comprise detailed design and executive design which allow a turnkey (i.e. complete) rail transport system to be provided.

Part of the configuration data needed for the PEP may be obtained from the BP. Indeed, the aim of high-level design is to verify transport system performances and costs and submit design choices to the approval of the customer (i.e. local administration) before proceeding to design in detail all components. In addition, orders for materials and products can be made at this stage, especially those that have long lead times of production.

In this context, our paper aims to provide an innovative approach in the design of a rail/metro system by implementing a single process from the bidding phase to the construction of the system. Hence, this paper is organised as follows: Section 2 describes the proposed framework of the Digital Pattern approach; Section 3 applies the proposed methodology to the case of the design of a metro system; finally, Section 4 presents the conclusions and outlines future potential for research.

\section{The proposed 'Digital Pattern' methodology}

Digital Pattern Product Development is an integrated computer system procedure which is designed to solve a number of critical issues in the bidding process and in the design of the signalling system and transportation solutions in order to reduce time to bidding and share company knowledge (tacit and explicit) worldwide. Indeed, the development of a Digital Pattern approach allows Ansaldo STS to implement a single process from the bidding phase to the construction of the rail system by organising, pooling and capitalising on company knowledge from different units.

In this context we may identify:

- a Digital Pattern product as a methodology for adapting a set of parameters already used in the past for similar projects which have become best practices;

- and, a Digital Pattern process as the methodology for integrating different processes.

The idea of optimising the bidding and design of technologies in the railway sector through a web platform has already been proposed elsewhere (see, for instance, [21] and [22]). However, these platforms did not encompass simulation tools. Conversely, the paradigm of the product digital pattern takes place in the DP system by integrating simulation models of rail traffic with wizard-based architecture. For a synopsis of the methodology adopted in the Digital Pattern approaches (i.e. DP product and DP process) (see Fig. 1). 


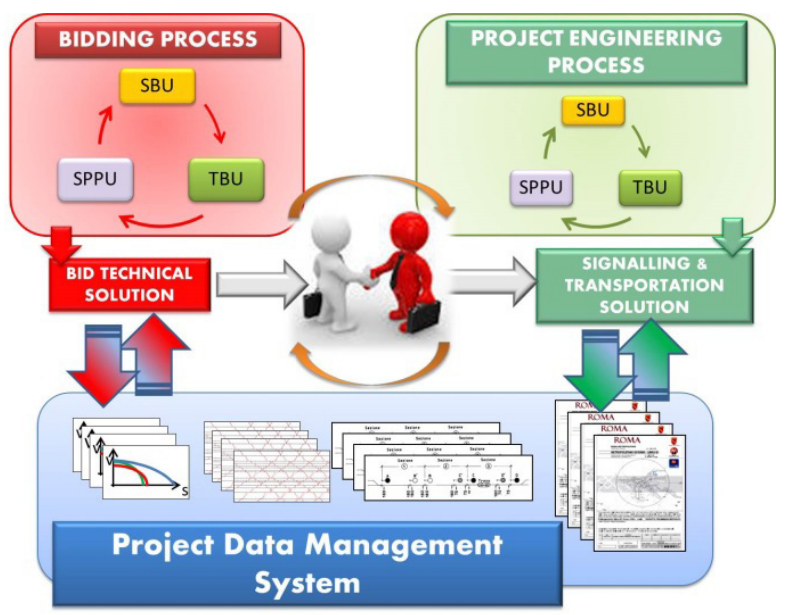

Figure 1: General framework of the Digital Pattern (DP) approach.

The DP framework was designed to meet the following general requirements:

(a) high processing performance; (b) data usability in time and in space;

(c) standard format of data for their import/export. Therefore, it is a platform created in a web environment that allows business staff:

- to create and transfer knowledge from a unit to another through the execution of software programs and applications on the platform (with access to and dynamic update of the Project Data Management System);

- to use a single format for data exchange;

- to collect, classify, organise and store all the input information;

- to use suitable servers for rapid access of staff to corporate knowledge.

\subsection{The three-layer architecture}

The Digital Pattern logical architecture generally comprises the following three levels:

- the frontend layer, which includes the interfaces that users are offered by the system to configure and manage the underlying services;

- the application layer, which represents the application logic and the logic of computing services system;

- the data layer that represents the logical structure that allows information to be kept about users, roles and profiles and the information required for the setup, execution and display of information managed by the system.

This architecture satisfies the requirements of flexibility and versatility expected from a system based on the Digital Pattern approach. Indeed, the flexibility requirement is satisfied by the ability to manage and/or scale each layer independently. Likewise, the versatility is ensured by the ability to make updates and/or changes also in the sub-layer of each level. 
The three logical levels are specified in this case as: the Digital Pattern Web Client (DPWC), the Digital Pattern Service Simulator (DPSS) and the Digital Pattern Data Base (DPDB).

\subsubsection{The Digital Pattern Web Client (DPWC)}

The DPWC is a component of the DP system responsible for the graphical interface for the user: through a unified interface, authorized users can perform different tasks according to their profile. The general environment is the web and allows users to access the various features that can be grouped into the following main areas:

- Design allows a new project to be created;

- Execution allows a new simulation process for existing projects to be set by changing some input parameters of the simulation models;

- Consultation allows data consultation only by authorised users;

- Monitoring targets users with an administrative role, and displays all the operations performed and recorded during the execution of DP applications;

- Export, enables the information to be exported in a standard format;

- Configuration targets only users with an administrative role and allows users to set the system configuration.

\subsubsection{The Digital Pattern Simulator Service (DPSS)}

The DPSS is a component of the DP system that includes the software components that run under the simulation models (the simulation tool suite). The simulation service essentially consists of a single Windows process called Host Simulator which in turn consists of the following software components:

- Data Access Management, responsible for the logic communication with the Digital Pattern database;

- Scheduler Management, responsible for logic scheduling simulation activities;

- Processing Simulator Thread Management, responsible for the execution of simulation processes.

\subsubsection{The Digital Pattern Data Base (DPDB)}

The DPDB is a component of the DP system responsible for the persistence of all data as well as those related to user access mechanisms and those related to the input/output simulation models. It is a database designed in SQL with a dynamic approach as it is progressively enriched with the output data of the simulation processes and new project settings.

Currently, the suite of simulation tools consists of a simulator prototype, classified as microscopic and synchronous, designed for subway traditional signalling system (ATP with continuous transmission by means of coded track circuits and discontinuous transmission by means of radio beacons). The Digital Pattern approach in the case of the ASTS can be described by Fig. 2. 


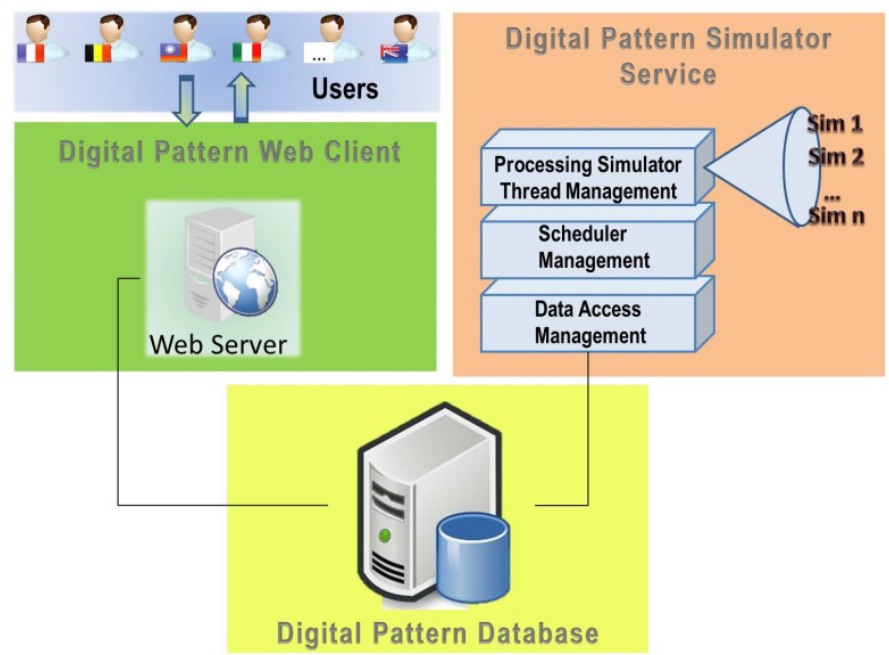

Figure 2: The framework of the Digital Pattern (DP) approach in the case of Ansaldo STS.

\section{An application to the design of a metro system}

The proposed methodology was applied in the case of the design of a metro system by adopting Ansaldo STS procedures. After the authentication procedure, the users can access the various functions depending on their role. In the case of creating a new project, the authorised user selects the type of project, such as 'Conventional Metro', where the type of project is defined by the technology used for the signalling system (for example, in the case of conventional metro lines the signalling system provides ATP by using coded track circuits). After selecting the type of project, the user can define infrastructure data (e.g. slopes, curve radius, maximum speeds) which may already be available in DPDB (only for existing lines) or imported from other design tools (e.g. Excel, Matlab, Autocad, etc.). Once the line has been set, the user can choose between a set of simulators available for the specific project type: each simulator performs a calculation procedure and therefore involves the insertion of specific input data and the generation of specific output data. In the Digital Pattern context, there are two available simulators:

- Simulator 1 (Sim1): Braking Curve Generation.

- Simulator 2 (Sim2): System performance verification.

Sim 1 consists of an algorithm for the generation of braking curves (expressed in terms of space-speed profiles). This first simulation procedure is essential for signalling system design because it allows the user to obtain braking distances. The output of the simulation using Sim1 consists of a data file for each 
calculated curve (service braking, emergency braking and rescue braking). It is important to stress that Sim1 calculates a set of braking curves (service, emergency and rescue) for each gradient of the track.

To run a simulation with $\mathrm{Sim} 1$ it is necessary to edit manually a series of parameters. All these parameters are classified into the following categories:

- General setting parameters;

- Rolling stock parameters;

- ATP parameters.

General setting parameters are related to the mode of execution of the simulation procedure, such as the sampling steps of the travel speed of the vehicle, the number of values in which the track is discretised, and so on. This data entry is performed via proper MMIs for the selected project type.

Rolling stock parameters are related to physical and performance parameters of the rolling stock (e.g. weight, length, number of traction units, traction force vs. speed profile and so on). This data entry is performed via MMIs suited to the selected project type.

Finally, ATP parameters are related to various equipment choices compatible with the type of signalling chosen. In particular, these sets of parameters include: those related to the continuous transmission equipment of the ATP system, those related to discontinuous transmission equipment of the ATP system, and those related to interface between the on-board application system and the braking system. However, all these parameters are delays related to transmission, data exchange between the equipment modules and processing of information data useful for ATP. Also this data entry is performed via MMIs suited to the selected project type.

The setting of the parameters related to the horizontal and vertical alignment of the track, rolling stock and ATP technology allows the simulation procedure to be run with Sim1. By varying one or more of the ATP parameters $N$ equipment simulations are obtained, with as many sets of braking curves as the number of modified parameters. The output of Sim1 is used in the design of the signalling system, in particular for the safe spacing of trains.

Once the braking curve has been obtained and before running Sim2 it is necessary to define in detail the dimensional characteristics of some components of the ATP systems, such as the size of the coded track circuits, the allocation of the signals in the line, and so on. In this phase the designer applies all his/her skills and experience and ASTS proprietary design tools.

After the design phase, the user can proceed with the use of Sim2. This is the simulator which allows the safe spacing of trains to be verified (as the length of block sections) in compliance with the time frequency of the line. The output of the simulation consists of a data file describing the kinematic parameters of the single vehicle movement (i.e. instantaneous speed, instantaneous acceleration, travelled space and time) including the dwell time spent at stations. Thus, Sim2 is used to verify the performance of the transport system in terms of train frequency and commercial speed. This can be done on the basis of the results of simulations carried out during manual design (e.g. sizing of track circuits based 
on the braking curves calculated with Sim1) or by using different values of input variables. Therefore, it can achieve $M$ different designs for the same system operating on the dimensional characteristics of the equipment components.

The proposed Digital Pattern methodology reveals many advantages which can be summarised as follows:

1. it is possible to access and modify existing project data anywhere and anytime by web access and also run simulations or create new projects. It depends on the type of user profile alone;

2. data sets can be imported from one project to another, provided that they belong to the same type of project;

3. General setting parameters, Rolling Stock parameters and ATP parameters may be derived from past projects which are stored in the Project Data Management System (i.e. the Digital Pattern System interfaces with PDMS);

4. since many of the data used for the design of the bidding technical solution are also necessary for project engineering, it is possible to reuse all data which are already stored so that all data entry phases can be avoided;

5. the final detail projects can be validated to check performance by using Digital Pattern simulators.

\section{Conclusions and research prospects}

In this paper we proposed a web platform to manage product development in the case of a multinational enterprise. The various phases of the industrial process (bidding, pre-design, design, production) require adoption of shared information. Hence knowledge management represents a key element which allows reductions in time and production costs.

The proposed Digital Pattern approach is based on a three-layer framework; the Digital Pattern Web Client (i.e. the user interface), the Digital Pattern Simulator Services (i.e. the simulation tool suite) and the Digital Pattern DataBase (i.e. the 'mind' and the 'memory' of the company). Finally, the innovative approach was applied in the case study of the design of the signalling system in the case of a traditional metro line, showing the benefits of adopting an integrated approach.

Future research could fruitfully address the number of participants and the complexity of the existing simulator by adopting the latest signalling technologies such as the ERTMS/ETCS and CBTC systems.

\section{Acknowledgement}

This paper is partially supported under research project PON - DIGITAL PATTERN grant no. PON01_01268. 


\section{References}

[1] Gallo, M., D’Acierno, L. \& Montella, B., A multimodal approach to bus frequency design. WIT Transactions on the Built Environment, 116, pp. 193-204, 2011.

[2] Gallo, M., Montella, B. \& D'Acierno, L., The transit network design problem with elastic demand and internalisation of external costs: An application to rail frequency optimisation. Transportation Research Part C, 19(6), pp. 1276-1305, 2011.

[3] Gallo, M., D'Acierno, L. \& Montella, B., A multilayer model to simulate cruising for parking in urban areas. Transport Policy, 18(5), pp. 735-744, 2011.

[4] D'Acierno, L., Ciccarelli, R., Montella, B. \& Gallo, M., A multimodal multiuser approach for analysing pricing policies in urban contexts. Journal of Applied Sciences, 11(4), pp. 599-609, 2011.

[5] Gallo, M., A fuel surcharge policy for reducing road traffic greenhouse gas emissions. Transport Policy, 18(2), pp. 413-424, 2011.

[6] Biggiero, L., The impact of transport management on local activities system: The role of limited traffic zones. WIT Transactions on the Built Environment, 138, 2014.

[7] Cascetta, E., Transportation systems analysis: models and applications. Springer: New York (NY), USA, 2009.

[8] D'Acierno, L., Gallo, M., Biggiero, L. \& Montella, B., Replanning public transport services in the case of budget reductions. WIT Transactions on the Built Environment, 138, 2014.

[9] D'Acierno, L., Gallo, M., Montella, B. \& Placido, A., Analysis of the interaction between travel demand and rail capacity constraints. WIT Transactions on the Built Environment, 128, pp. 197-207, 2012.

[10] D'Acierno, L., Gallo, M., Montella, B. \& Placido, A., The definition of a model framework for managing rail systems in the case of breakdowns. Proc. of the 16th IEEE Conference on Intelligent Transportation Systems (ITSC), pp. 1059-1064, 2013.

[11] Ercolani, M., Placido, A., D'Acierno, L. and Montella, B., The use of microsimulation models for the planning and management of metro systems. WIT Transactions on the Built Environment, 135, 2014.

[12] Corman, F., D'Ariano, A., Hansen, I.A. \& Pacciarelli, D., Optimal multiclass rescheduling of railway traffic. Journal of Rail Transport Planning \& Management, 1(1), pp. 14-24, 2011.

[13] van den Boom, T.J.J., Kersbergen, B. \& De Schutter, B., Structured modeling, analysis, and control of complex railway operations. Proc. of the 51st IEEE Conference on Decision and Control, pp. 7366-7371, 2012.

[14] Hansen, I.A., Goverde, R.M.P. \& van der Meer, D.J., Online train delay recognition and running time prediction. Proc. of the 13th International IEEE Conference on Intelligent Transportation Systems (ITSC), pp. 1783-1788, 2010. 
[15] Goverde, R.M.P., A delay propagation algorithm for large-scale railway traffic networks. Transportation Research Part C, 18(3), pp. 269-287, 2010.

[16] Goverde, R.M.P. \& Meng, L., Advanced monitoring and management information of railway operations. Journal of Rail Transport Planning \& Management, 1(2), pp. 69-79, 2011.

[17] Wang, Y., De Schutter, B., Ning, B., Groot, N. \& van den Boom, T.J.J., Optimal trajectory planning for trains using mixed integer linear programming. Proc. of the 14th International IEEE Conference on Intelligent Transportation Systems (ITSC), pp. 1598-1603, 2011.

[18] Corapi, G., Sanzari, D., De Martinis, V., D’Acierno, L. \& Montella, B., A simulation-based approach for evaluating train operating costs under different signalling systems. WIT Transactions on the Built Environment, 130, pp. 149-161, 2013.

[19] De Martinis, V., Gallo, M. \& D'Acierno, L., Estimating the benefits of energy-efficient train driving strategies: a model calibration with real data. WIT Transactions on the Built Environment, 130, pp. 201-211, 2013.

[20] Corapi, G, De Martinis, V., Placido, A. \& De Luca, G., Impacts of Energy Saving Strategies (ESSs) on rail services and related effects on travel demand. WIT Transactions on the Built Environment, 135, 2014.

[21] Penciuc, D., Abel, M. \& Van Den Abeele, D., Modelling and architecture of a workspace for tacit knowledge management in railway transport. Proc. of the 22nd International Conference on Tools with Artificial Intelligence (ICTAI), pp. 356-357, 2010.

[22] Penciuc D., Abel M-H., Van Den Abeele D., How can knowledge management improve the tendering process in railway transport. Proc. of the 5th International Conference on Computer Supported Cooperative Work in Design, pp. 813-818, 2011. 